Article

\title{
$S U(2)$ Symmetry of Qubit States and Heisenberg-Weyl Symmetry of Systems with Continuous Variables in the Probability Representation of Quantum Mechanics
}

\author{
Peter Adam ${ }^{1,2}$, Vladimir A. Andreev ${ }^{3}$, Margarita A. Man'ko ${ }^{3, *}$ and Vladimir I. Man'ko ${ }^{3,4}$ \\ and Matyas Mechler ${ }^{2}$ \\ 1 Institute for Solid State Physics and Optics, Wigner Research Center for Physics, P.O. Box 49, \\ H-1525 Budapest, Hungary; adam.peter@wigner.mta.hu \\ 2 Institute of Physics, University of Pécs, Ifjúság útja 6, H-7624 Pécs, Hungary; mechler@fizika.ttk.pte.hu \\ 3 Lebedev Physical Institute, Leninskii Prospect 53, Moscow 119991, Russia; andrvlad@yandex.ru (V.A.A.); \\ mankovi@lebedev.ru or manko.vi@phystech.edu (V.I.M.) \\ 4 Moscow Institute of Physics and Technology, State University, Institutskii per. 9, Dolgoprudnyi, \\ Moscow Region 141700, Russia \\ * Correspondence: mankoma@lebedev.ru
}

Received: 18 April 2020; Accepted: 10 May 2020; Published: 2 July 2020

\begin{abstract}
In view of the probabilistic quantizer-dequantizer operators introduced, the qubit states (spin-1/2 particle states, two-level atom states) realizing the irreducible representation of the $S U$ (2) symmetry group are identified with probability distributions (including the conditional ones) of classical-like dichotomic random variables. The dichotomic random variables are spin-1/2 particle projections $m= \pm 1 / 2$ onto three perpendicular directions in the space. The invertible maps of qubit density operators onto fair probability distributions are constructed. In the suggested probability representation of quantum states, the Schrödinger and von Neumann equations for the state vectors and density operators are presented in explicit forms of the linear classical-like kinetic equations for the probability distributions of random variables. The star-product and quantizer-dequantizer formalisms are used to study the qubit properties; such formalisms are discussed for photon tomographic probability distribution and its correspondence to the Heisenberg-Weyl symmetry properties.
\end{abstract}

Keywords: quantum tomography; probability representation; quantizer-dequantizer; qubit

\section{Introduction}

In conventional formulation of quantum mechanics, the states of quantum systems are identified either with wave functions [1,2] and state vectors [3] (in the case of pure states) or with density matrices [4] and density operators [5] (in the case of mixed states). The unitary time evolution of quantum states is described by the Schrödinger equation for the wave function and the von Neumann equation for the density operator. An important role in the description of quantum states is played by the symmetry properties of quantum systems. They are determined by the operators of Lie groups (or Lie algebras) associated with Hamiltonian operators of the system. The solutions of the Schrödinger equation (wave functions) realize the irreducible representations of the symmetry groups. For spin systems, the rotation symmetry group $O(3)$ and $S U(2)$ group and their representations provide the description of wave functions and Hamiltonian spectra, as well as the unitary evolution of wave functions and density matrices. 
Over recent decades, the probability representation of quantum states, where the states are identified with fair probability distributions of random variables, was suggested and elaborated (see, for example, the review [6]); it is connected with the approach based on quantum tomography of photon states [7] (see also [8]). The state quantum tomograms, being the tomographic-probability distributions of photon quadratures, are measured experimentally and, by means of its Radon transform [9], the Wigner function of the photon state [10,11] is reconstructed. Since the Wigner function and the density matrix are completely determined by the tomographic-probability distribution, it was suggested in [12] to identify any quantum state with the probability distribution as a primary object.

The Schrödinger equation for the photon tomogram was written in the form of a kinetic equation for the probability distribution [13-15]. An analogous representation of spin states by probability distributions was discussed in [16-20] and the connection of quantum states with probabilities was considered in [21]. In view of the introduced probability representation of quantum states, it is worth clarifying the role of symmetries in the probability representation of quantum mechanics.

The other aspect of quantum states, as well as quantum observables and their relation to classical mechanics, is associated with the quantization procedure. Physical observables, which conventionally are identified with Hermitian operators acting in the Hilbert space of system states are mapped onto functions called symbols of operators, e.g., in the phase space. The quantum nature of the observables is described by a specific rule of products of these functions (called the star-product), which is noncommutative but associative. The associative product is determined by structure constants satisfying the equations of associativity corresponding to the associativity condition of the product of operators identified with physical observables. The structure constants of the associative product determine the Lie algebra structure constants. Thus, the symmetries (Lie algebras, Lie groups) of quantum system Hamiltonians are connected with the star-product structure constants.

One of the goals of our work is to discuss the quantization procedure presented within the framework of star-product formalism relating this approach to the probability representation of quantum mechanics. We introduce the approach to Lie algebras and corresponding symmetries of systems with the star-product structure constants associated with quantization procedure providing the description of states by probability distributions.

The aim of our work is to point out the role of the $S U(2)$ symmetry group and its fundamental two-dimensional irreducible representation used to describe the quantum state of the spin-1/2 systems (also qubits, two-level atoms) in the context of the probability representation of quantum mechanics [12-17] and the star-product quantization formalism [22,23]. In addition, we consider the Heisenberg-Weyl symmetry group for systems with continuous variables like oscillator. The pure states of spin-1/2 systems are identified with Pauli spinors, which provide the basis of two-dimensional unitary irreducible representation of the $S U(2)$ group used to explain the behavior of the spin-1/2 particle related to rotations connected with the $O(3)$ symmetry group. The mixed states of the spin- $1 / 2$ particle are identified with the density $2 \times 2$-matrices, which are Hermitian matrices with unit trace and nonzero eigenvalues.

Recently $[16,17,24,25]$, the possibility to construct the invertible map of the density matrices onto probability distributions of classical-like random variables was suggested. This possibility to construct such probability representation of quantum states provides a generalization of the quasidistribution phase-space representations [26-31] (including the case of systems with discrete variables), where the states are described by the functions on the system phase space similar to classical probability distributions but, due to the Heisenberg uncertainty relations [32] and Schrödinger-Robertson uncertainty relations [33,34], the functions cannot be the probability distributions of conjugate variables like the position and momentum. For systems with discrete spin variables, the corresponding functions like Wigner functions were studied in [31,35]. An analog of such Wigner functions is the symbol of density operator in the spin-projection mean-value representation, which is the description of the state by the Bloch parameter. 
One can use other methods of quantization to construct the formalism of quantum mechanics, in view of the application of associative star-product, where the product of functions is associative but not commutative. Such a product can be introduced by applying recently developed method $[22,23,36]$ of the quantizer-dequantizer operators. The quantizer operators were used $[30,31,35]$ to construct quasidistributions describing the quantum system states, and the pairs of quantizer-dequantizer operators were found to construct symplectic tomographic probability distributions identified with the system states with continuous variables [37]. The associative product is given if the structure constants of the product are found; these constants satisfy nonlinear associativity relations.

In this study, we concentrate on the product (quantization procedure) namely for the case of probabilistic formulation of states on examples of the qubit system and the system with continuous variables like oscillator. The quantum system dynamics is closely related with the symmetry of systems. We consider systems with Hermitian Hamiltonian dynamics and unitary evolution [38]. It is worth noting that there exist the models with non-Hermitian dynamics [39-42].

This paper is organized as follows.

In Section 2, we review a generic formalism of the quantization procedure based on introducing pairs of quantizer-dequantizer operators determining the structure constants of the associative product of functions. In Section 3, we discuss the Lie algebra structure constants and their connection with the associative product structure constants. In Section 4, we consider the $S U(2)$ symmetry of spin-1/2 states in the quantization picture based on the probability representation of these states. In Section 5 , the von Neumann evolution equation for density matrix is given in vector form. In Section 6, we present the von Neumann equation for the unitary evolution of the spin-1/2 state density matrix in the probability representation. In Sectionn 7 , the $O(3)$ rotation group of transforms of probabilities and spin-projection mean values are discussed. Unitary transforms of quantizer-dequantizer operators are given in Section 8. The systems with continuous variables like, for example, photons or harmonic oscillator, are considered in Section 9 using the Heisenberg-Weyl formalism, and our conclusions are presented in Section 10.

\section{Quantizer-Dequantizer Operators and Structure Constants of Associative Products}

In this section, we consider a Hilbert space $\mathcal{H}$ with operators $\hat{U}(x)$ and $\hat{D}(x)$ acting in this Hilbert space, where $x=\left(x_{1}, x_{2}, \ldots, x_{n}\right)$ and $x_{j}$ are real numbers, either continuous or discrete ones. The operators $\hat{U}(x)$ and $\hat{D}(x)$ are called the dequantizer and quantizer, respectively [22,23]. They satisfy the following conditions: For an arbitrary operator $\hat{A}$ acting in the Hilbert space, we construct the function $f_{A}(x)$ called the symbol of operator

$$
f_{A}(x)=\operatorname{Tr}(\hat{A} \hat{U}(x)) .
$$

Using the quantizer operator $\hat{D}(x)$, we reconstruct the operator $\hat{A}$, in view of the relation

$$
\hat{A}=\int f_{A}(x) \hat{D}(x) d x
$$

if some parameters $x_{j}$ are discrete ones, the integral (2) is replaced by the corresponding sum over discrete parameters.

Relations (1) and (2) are self-consistent if the quantizer-dequantizer operators satisfy the condition

$$
\operatorname{Tr}(\hat{A} \hat{U}(x))=\int \operatorname{Tr}\left(\hat{A} \hat{U}\left(x^{\prime}\right)\right) \operatorname{Tr}\left(\hat{U}(x) \hat{D}\left(x^{\prime}\right)\right) d x^{\prime}
$$

for any operator $\hat{A}$ in the Hilbert space $\mathcal{H}$. Relation (3) is valid if there exists the equality

$$
\operatorname{Tr}\left(\hat{U}\left(x^{\prime}\right) \hat{D}(x)\right)=\delta\left(x-x^{\prime}\right) ;
$$


where $\delta\left(x-x^{\prime}\right)$ is the reproducing kernel. Equality (4) is not the necessary condition for relation (3) but it is the sufficient condition.

We point out an important partial case of choosing the pair of quantizer-dequantizer operators, for which the function $f_{A}(x)$, being the symbol of operator $\hat{A}$, has the following properties:

If the operator $\hat{A}$ is taken to be a density operator $\hat{\rho}$, i.e., $\hat{\rho}^{\dagger}=\hat{\rho}, \operatorname{Tr} \hat{\rho}=1$, and $\hat{\rho}$ has nonnegative eigenvalues, the function $f_{\rho}(x) \geq 0$ and it is normalized, i.e.,

$$
\int f_{\rho}(x) d[x]=1, \quad d[x] \equiv d x_{1} d x_{2} \cdots d x_{j} .
$$

The integral over the parameters $[x]$ in (5) means that we integrate (or sum) only over the first part of parameters $x_{1}, x_{2}, \ldots, x_{j} ; j<n$, and parameters $x_{j+1}, x_{j+2}, \ldots, x_{n}$ are considered as fixed parameters, while the indices $j$ are determined according the physical properties of the system under consideration (for example, in Section 9, $x=x_{1}, x_{2}, x_{2}$ and $j=1$ ). This property means that, in this case, the function $f_{\rho}(x)$ can be interpreted as a conditional probability distribution, and we introduce the notation

$$
f_{\rho}(x)=P\left(x_{1}, x_{2}, \ldots, x_{j} \mid x_{j+1}, x_{j+2}, \ldots, x_{n}\right) .
$$

For this case, parameters $x_{1}, x_{2}, \ldots, x_{n}$ can be interpreted as classical-like random variables.

In view of Bayes' formula [43], one can introduce the joint probability distribution $\Pi(x)$ of $n$ random variables $x_{1}, x_{2}, \ldots, x_{n}$ written as

$$
\Pi(x)=f_{\rho}(x) \mathcal{P}\left(x_{j+1}, x_{j+2}, \ldots, x_{n}\right),
$$

where the function $\mathcal{P}\left(x_{j+1}, x_{j+2}, \ldots, x_{n}\right) \geq 0$ and

$$
\int \mathcal{P}\left(x_{j+1}, x_{j+2}, \ldots, x_{n}\right) d x_{j+1} d x_{j+2} \cdots d x_{n}=1 .
$$

If some of the variables in (8) are discrete ones, the integral over these variables is replaced by the corresponding sum over these variables.

The possibility to find the quantizer-dequantizer pair, $\hat{D}(x)$ and $\hat{U}(x)$, with properties (5)-(8) means that one can construct an invertible map of density operators of quantum states onto probability distributions. This possibility realized in [22] provides the formulation of quantum mechanics in the form of classical-like statistics, where quantum states are identified with probability distributions obeying classical-like kinetic equations. In this paper, we consider particular examples of the above approach for qubit states and photon states, where the number of random variable is not larger than three.

\section{Structure Constants of Lie Algebras and Their Relation to Associative Product Structure Constants}

The product of operators $\hat{A} \hat{B}$ has the symbol, which is the function $f_{A B}(x)$ of the form

$$
f_{A B}(x)=\operatorname{Tr}(\hat{A} \hat{B} \hat{U}(x))=\int f_{A}\left(x_{1}\right) f_{B}\left(x_{2}\right) \operatorname{Tr}\left(\hat{D}\left(x_{1}\right) \hat{D}\left(x_{2}\right) \hat{U}(x)\right) d x_{1} d x_{2} .
$$

Functions

$$
K\left(x_{1}, x_{2}, x\right)=\operatorname{Tr}\left(\hat{D}\left(x_{1}\right) \hat{D}\left(x_{2}\right) \hat{U}(x)\right)
$$

are called the structure constants of the associative product or the star-product of functions $f_{A}(x)$ and $f_{B}(x)$ defined as

$$
\left(f_{A} \star f_{B}\right)(x)=f_{A B}(x)
$$


This product is associative, i.e.,

$$
\left(\left(f_{A} \star f_{B}\right) \star f_{C}\right)(x)=\left(f_{A} \star\left(f_{B} \star f_{C}\right)\right)(x),
$$

since the product of operators is associative, i.e., $(\hat{A} \hat{B}) \hat{C}=\hat{A}(\hat{B} \hat{C})$. The associativity condition provides nonlinear integral relation for the structure constants

$$
\int d z K\left(x_{1}, x_{2}, z\right) K\left(z, x_{3}, x\right)=\int d z K\left(x_{2}, x_{3}, z\right) K\left(x_{1}, z, x\right) .
$$

If variables $x_{1}, x_{2}, x_{3}, x_{4}$, and $z$ are discrete variables and functions $K\left(x_{1}, x_{2}, z\right)$ are denoted as $K\left(x_{1}=i, x_{2}=j, z=k\right) \equiv C_{i j}^{k} ; i, j, k=1,2, \ldots, N$, Equation (13) takes the form

$$
\sum_{k=1}^{N}\left(C_{i j}^{k} C_{k m}^{n}-C_{j m}^{k} C_{i k}^{n}\right)=0
$$

The solutions to Equations (13) and (14) provide the structure constants for associative algebras either in the case of functions of continuous variables (the case of (13)) or in the case of functions of discrete variables (the case of (14)).

If one uses antisymmetrized structure constants (10), one has the structure constants $L\left(x_{1}, x_{2}, z\right)$ of Lie algebras determining the Lie products of functions. The constants satisfying Jacobi equation are

$$
L\left(x_{1}, x_{2}, z\right)=K\left(x_{1}, x_{2}, z\right)-K\left(x_{2}, x_{1}, z\right) .
$$

For discrete variables, we have the finite or infinite Lie algebra with structure constants

$$
\ell_{i j}^{k}=C_{i j}^{k}-C_{j i}^{k}
$$

Thus, the symmetry of systems described by Lie groups, e.g., $O(4,2)$ symmetry of the hydrogen atom [44-47] can be associated with the corresponding associative algebras, that provides the possibility to relate the spectra of system's Hamiltonians with the associative products of symbols of physical-observable operators. The symmetrized structure constants are the structure constants of Jordan product of the functions. The constants are

$$
\begin{array}{r}
s_{i j}^{k}=C_{i j}^{k}+C_{j i}^{k} \quad \text { for discrete variables, } \\
S\left(x_{1}, x_{2}, z\right)=K\left(x_{1}, x_{2}, x\right)+K\left(x_{2}, x_{1}, x\right) \quad \text { for continuous variables. }
\end{array}
$$

We point out that the formalism of quantizer-dequantizer operators can be used to describe the Lie group symmetry of physical systems, if the associative product structure constants are found in explicit form.

\section{4. $S U(2)$ Symmetry and the Probability Representation of Spin-1/2 States}

We consider a construction of the probability representation of quantum states on a simplest example of spin-1/2 states (qubit states), which are connected with unitary irreducible representation of the $S U(2)$ Lie group (and Lie algebra $s u(2)$ ).

For this, first we recall that the pure state of spin-1/2 particle $|\psi\rangle$ is a vector in a two-dimensional Hilbert space $\mathcal{H}$ with complex components $\psi_{k} ; k=1,2$. The vector is normalized $\sum_{k=1}^{2}\left|\psi_{k}\right|^{2}=1$ and depends on three real parameters. The density $2 \times 2$-matrix $\rho_{k j}=\psi_{k} \psi_{j}^{*}$ of the pure state $|\psi\rangle$ is Hermitian $2 \times 2$-matrix with the properties $\rho^{\dagger}=\rho, \operatorname{Tr} \rho=1$, and two eigenvalues $\lambda_{1}=1$ and $\lambda_{2}=0$. This matrix (projector) satisfies the condition $\rho^{2}=\rho$, i.e., $\operatorname{Tr} \rho^{2}=\operatorname{Tr} \rho=1$, and this means that, in view of the gauge invariance of quantum states, the above state vector can be presented in the form [48-50] 


$$
\psi_{1}=\sqrt{p_{3}} \quad \text { and } \quad \psi_{2}=\sqrt{\left(1-p_{3}\right)} e^{i \varphi}
$$

where

$$
\begin{array}{ll}
\cos \varphi=\frac{p_{1}-1 / 2}{\sqrt{p_{3}\left(1-p_{3}\right)}}, & \sin \varphi=\frac{p_{2}-1 / 2}{\sqrt{p_{3}\left(1-p_{3}\right)}}, \\
0 \leq p_{1}, p_{2}, p_{3} \leq 1, & \left(p_{1}-1 / 2\right)^{2}+\left(p_{2}-1 / 2\right)^{2}=p_{3}\left(1-p_{3}\right) .
\end{array}
$$

Numbers $p_{1}, p_{2}$, and $p_{3}$ can be expressed as follows:

$$
p_{1}=\frac{1}{2}+\frac{1}{2} \sin \theta \cos \varphi, \quad p_{2}=\frac{1}{2}+\frac{1}{2} \sin \theta \sin \varphi, \quad p_{3}=\frac{1}{2}+\frac{1}{2} \cos \theta ; \quad 0 \leq \theta \leq \pi ; \quad 0 \leq \varphi \leq 2 \pi .
$$

One should emphasize that $p_{1}, p_{2}$, and $p_{3}$ are used to pass from a sphere of radius 1 centered at the origin to a sphere of radius $1 / 2$ centered at $(1 / 2,1 / 2,1 / 2)$. In such a way, all coordinates are positive and smaller or equal to 1 . In (19), $p_{3}=\cos \theta / 2$ and $\left(1-p_{3}\right)=\sin \theta / 2$.

The density matrix of the pure state depends on real parameters $p_{1}, p_{2}$, and $p_{3}$, which are interpreted as the probabilities of spin projections $m=+1 / 2$ on the $x, y$, and $z$ directions, respectively. Thus, the identification of spin-1/2 pure states with the Pauli spinor $|\psi\rangle$ is equivalent to the identification of the state with three probability distributions $\left(p_{1}, 1-p_{1}\right),\left(p_{2}, 1-p_{2}\right)$, and $\left(p_{3}, 1-p_{3}\right)$ of dichotomic random variables.

In the case of mixed states, the density matrix depends on three real parameters $p_{1}, p_{2}$, and $p_{3}$, which satisfy the inequality

$$
\left(p_{1}-1 / 2\right)^{2}+\left(p_{2}-1 / 2\right)^{2}+\left(p_{3}-1 / 2\right)^{2} \leq 1 / 4
$$

for the pure states, this inequality converts into the equality.

We formulated above results using the map of the density matrix $\rho_{k j}$ onto the vector $|R\rangle$ with four components $R_{\alpha} ; \alpha=1,2,3,4$. The map is given by the following rule.

The pairs of numbers $k$ and $j$, where $k, j=1,2$, are replaced by one number, which takes values $\alpha=1,2,3,4$, namely, $1,1 \Longleftrightarrow 1, \quad 1,2 \Longleftrightarrow 2, \quad 2,1 \Longleftrightarrow 3, \quad 2,2 \Longleftrightarrow 4$.

Any state density matrix $\rho$ is identified with a vector

$$
|R\rangle=\left(\begin{array}{c}
\rho_{11} \\
\rho_{12} \\
\rho_{21} \\
\rho_{22}
\end{array}\right), \quad \text { such that } \quad\langle R \mid R\rangle=\sum_{j, k=1}^{2}\left|\rho_{j k}\right|^{2} .
$$

For pure states, the vector reads

$$
\left|R_{\psi}\right\rangle=\left(\begin{array}{c}
p_{3} \\
\sqrt{p_{3}\left(1-p_{3}\right)} e^{-i \varphi} \\
\sqrt{p_{3}\left(1-p_{3}\right)} e^{i \varphi} \\
1-p_{3}
\end{array}\right) ; \quad e^{i \varphi}=\frac{\left(p_{1}-1 / 2\right)+i\left(p_{2}-1 / 2\right)}{\sqrt{p_{3}\left(1-p_{3}\right)}} .
$$

We have $\left\langle R_{0}\right|=(1,0,0,1)$, where $\left\langle R_{0} \mid R_{\psi}\right\rangle=1$ and $\left|R_{\psi}\right\rangle$ is the state vector (22).

In (21), the components of four-vector $|R\rangle$ obey the constraints, corresponding to the properties: $\operatorname{Im} \rho_{k k}=0, \quad \sum_{k} \rho_{k k}=1$, and $\rho_{k j}=\rho_{j k}^{*}$. One has $\left\langle R_{\psi} \mid R_{\psi}\right\rangle=1$. The Hermitian $4 \times 4$-matrix $|R\rangle\langle R|$ has 16 matrix elements $R_{\alpha \beta}$ in the notation using the components $R_{\alpha}$ and $R_{\beta}$ of the vector $|R\rangle ; \alpha, \beta=1,2,3,4$ applied in (21). For pure states, the $4 \times 4$-matrix $\left|R_{\psi}\right\rangle\left\langle R_{\psi}\right|$ depends on three parameters $p_{1}, p_{2}$, and $p_{3}$ satisfying equality in (20). 
We present the density $2 \times 2$-matrix $\rho$ [24]

$$
\rho=\left(\begin{array}{cc}
p_{3} & \left(p_{1}-1 / 2\right)-i\left(p_{2}-1 / 2\right) \\
\left(p_{1}-1 / 2\right)+i\left(p_{2}-1 / 2\right) & 1-p_{3}
\end{array}\right)
$$

in the vector form (21)

$$
|R\rangle=\left(\begin{array}{c}
p_{3} \\
\left(p_{1}-1 / 2\right)-i\left(p_{2}-1 / 2\right) \\
\left(p_{1}-1 / 2\right)+i\left(p_{2}-1 / 2\right) \\
1-p_{3}
\end{array}\right)
$$

One sees that

$$
\langle R \mid R\rangle=2\left[p_{1}^{2}+p_{2}^{2}+p_{3}^{2}-p_{1}-p_{2}-p_{3}+1\right]
$$

We have the following form of the $4 \times 4$ matrix $|R\rangle\langle R|=\left(\begin{array}{cc}A & C \\ B & D\end{array}\right)$, where

$$
\begin{aligned}
A & =\left(\begin{array}{cc}
p_{3}^{2} & p_{3}\left[\left(p_{1}-1 / 2\right)+i\left(p_{2}-1 / 2\right)\right] \\
{\left[\left(p_{1}-1 / 2\right)-i\left(p_{2}-1 / 2\right)\right] p_{3}} & \left(p_{1}-1 / 2\right)^{2}+\left(p_{2}-1 / 2\right)^{2}
\end{array}\right), \\
B & =\left(\begin{array}{cc}
{\left[\left(p_{1}-1 / 2\right)-i\left(p_{2}-1 / 2\right)\right] p_{3}} & {\left[\left(p_{1}-1 / 2\right)+i\left(p_{2}-1 / 2\right)\right]^{2}} \\
\left(1-p_{3}\right) p_{3} & \left(1-p_{3}\right)\left[\left(p_{1}-1 / 2\right)+i\left(p_{2}-1 / 2\right)\right]
\end{array}\right), \\
C & =\left(\begin{array}{cc}
p_{3}\left[\left(p_{1}-1 / 2\right)-i\left(p_{2}-1 / 2\right)\right] & p_{3}\left(1-p_{3}\right) \\
{\left[\left(p_{1}-1 / 2\right)-i\left(p_{2}-1 / 2\right)\right]^{2}} & {\left[\left(p_{1}-1 / 2\right)-i\left(p_{2}-1 / 2\right)\right]\left(1-p_{3}\right)}
\end{array}\right), \\
D & =\left(\begin{array}{cc}
\left(p_{1}-1 / 2\right)^{2}+\left(p_{2}-1 / 2\right)^{2} & {\left[\left(p_{1}-1 / 2\right)+i\left(p_{2}-1 / 2\right)\right]\left(1-p_{3}\right)} \\
\left(1-p_{3}\right)\left[\left(p_{1}-1 / 2\right)-i\left(p_{2}-1 / 2\right)\right] & \left(1-p_{3}\right)^{2}
\end{array}\right) .
\end{aligned}
$$

One can check that $\left(\left|R_{\psi}\right\rangle\left\langle R_{\psi}\right|\right)^{2}=\left|R_{\psi}\right\rangle\left\langle R_{\psi}\right|$.

Thus, we see that the pure state of qubit determined by Pauli spinor $|\psi\rangle$ determines the pure state of the ququart with the density $4 \times 4$-matrix $\left|R_{\psi}\right\rangle\left\langle R_{\psi}\right|$. This ququart pure state is identified with the same three probability distributions of dichotomic random variables, which are identified with the qubit state. Continuing this procedure of vectorizing matrices, one can construct specific states of qudits with $N_{n}=2^{2^{n}} ; n=1,2,3, \ldots$

Thus, we showed that the probabilities $p_{1}, p_{2}$, and $p_{3}$, determining the density matrix of the qubit pure state, also determine the infinite set of density matrices of pure states of qudits.

As it is known, for matrices $A$ and $B$, the trace of their product $\operatorname{Tr}\left(A^{\dagger} B\right)=\langle A \mid B\rangle$, where $|A\rangle$ and $|B\rangle$ are vectors written according to (21). In view of this fact [51], one can formulate the matrix properties using the vectors corresponding to these matrices. The representation of matrices as sums of other matrices $A=\sum_{k=1}^{n} c_{k} A_{k}$ can be presented in the corresponding vector forms: $|A\rangle=\sum_{k=1}^{n} c_{k}\left|A_{k}\right\rangle$. Thus, we can use the vectors $\left|A_{k}\right\rangle$, which form the basis in the linear space, with $n=4$.

In the case of $S U(2)$-symmetry, the Pauli matrices $\sigma_{1}=\left(\begin{array}{ll}0 & 1 \\ 1 & 0\end{array}\right), \quad \sigma_{2}=\left(\begin{array}{cc}0 & -i \\ i & 0\end{array}\right)$, and $\sigma_{3}=\left(\begin{array}{cc}1 & 0 \\ 0 & -1\end{array}\right)$ realizing the $s u(2)$ Lie algebra and the unity matrix $\sigma_{0}=\left(\begin{array}{ll}1 & 0 \\ 0 & 1\end{array}\right)$ form this basis. In the vector form, we have the corresponding orthonormal basis

$$
\left|\Sigma_{1}\right\rangle=\left(\begin{array}{c}
0 \\
1 / \sqrt{2} \\
1 / \sqrt{2} \\
0
\end{array}\right), \quad\left|\Sigma_{2}\right\rangle=\left(\begin{array}{c}
0 \\
-i / \sqrt{2} \\
i / \sqrt{2} \\
0
\end{array}\right), \quad\left|\Sigma_{3}\right\rangle=\left(\begin{array}{c}
1 / \sqrt{2} \\
0 \\
0 \\
-1 / \sqrt{2}
\end{array}\right), \quad\left|\Sigma_{4}\right\rangle=\left(\begin{array}{c}
1 / \sqrt{2} \\
0 \\
0 \\
1 / \sqrt{2}
\end{array}\right)
$$


with $\left\langle\Sigma_{\alpha} \mid \Sigma_{\beta}\right\rangle=\delta_{\alpha \beta}$.

An arbitrary matrix $A=\left(\begin{array}{ll}A_{11} & A_{12} \\ A_{21} & A_{22}\end{array}\right)$ presented in vector form (21) can be decomposed, using basis vectors (27) and the notation $\widetilde{A}_{1}=A_{11}, \widetilde{A}_{2}=A_{12}, \widetilde{A}_{3}=A_{21}$, and $\widetilde{A}_{4}=A_{22}$, as follows:

$$
|A\rangle=\left(\begin{array}{c}
\widetilde{A}_{1} \\
\widetilde{A}_{2} \\
\widetilde{A}_{3} \\
\widetilde{A}_{4}
\end{array}\right)=\sum_{\alpha=1}^{4}\left\langle\Sigma_{\alpha} \mid A\right\rangle\left|\Sigma_{\alpha}\right\rangle
$$

There exist other normalized basis vectors useful for constructing the probability representation of spin- $1 / 2$ states, such as

$$
\left|U_{1}\right\rangle=\frac{1}{2}\left(\begin{array}{l}
1 \\
1 \\
1 \\
1
\end{array}\right), \quad\left|U_{2}\right\rangle=\frac{1}{2}\left(\begin{array}{c}
1 \\
-i \\
i \\
1
\end{array}\right), \quad\left|U_{3}\right\rangle=\left(\begin{array}{l}
1 \\
0 \\
0 \\
0
\end{array}\right), \quad\left|U_{4}\right\rangle=\left(\begin{array}{l}
0 \\
0 \\
0 \\
1
\end{array}\right)
$$

They correspond to the density matrices

$$
\rho_{1}=\frac{1}{2}\left(\begin{array}{ll}
1 & 1 \\
1 & 1
\end{array}\right), \quad \rho_{2}=\frac{1}{2}\left(\begin{array}{cc}
1 & -i \\
i & 1
\end{array}\right), \quad \rho_{3}=\left(\begin{array}{ll}
1 & 0 \\
0 & 0
\end{array}\right), \quad \rho_{4}=\left(\begin{array}{ll}
0 & 0 \\
0 & 1
\end{array}\right) .
$$

In view of Born's rule, for an arbitrary density matrix $\rho$, the numbers $\operatorname{Tr} \rho \rho_{k}=p_{k}$ are probabilities to get the properties of states $\rho_{k}$, if one studies these properties in the state $\rho$. Since vectors (29) form the basis, one has the probability representation of the state $\rho$, which is identical with probabilities $p_{k}$.

We have properties relating bases (27) and (29). Applying the transforms

$$
\begin{array}{ll}
\left|U_{1}\right\rangle=\frac{1}{\sqrt{2}}\left(\left|\Sigma_{1}\right\rangle+\left|\Sigma_{4}\right\rangle\right), & \left|U_{2}\right\rangle=\frac{1}{\sqrt{2}}\left(\left|\Sigma_{2}\right\rangle+\left|\Sigma_{4}\right\rangle\right), \\
\left|U_{3}\right\rangle=\frac{1}{\sqrt{2}}\left(\left|\Sigma_{4}\right\rangle+\left|\Sigma_{3}\right\rangle\right), & \left|U_{4}\right\rangle=\frac{1}{\sqrt{2}}\left(\left|\Sigma_{4}\right\rangle-\left|\Sigma_{3}\right\rangle\right),
\end{array}
$$

we arrive at

$$
\begin{array}{ll}
\left|\Sigma_{1}\right\rangle=\sqrt{2}\left|U_{1}\right\rangle-\frac{1}{\sqrt{2}}\left(\left|U_{3}\right\rangle+\left|U_{4}\right\rangle\right), & \left|\Sigma_{2}\right\rangle=\sqrt{2}\left|U_{3}\right\rangle-\frac{1}{\sqrt{2}}\left(\left|U_{3}\right\rangle+\left|U_{4}\right\rangle\right) . \\
\left|\Sigma_{3}\right\rangle=\frac{1}{\sqrt{2}}\left(\left|U_{3}\right\rangle-\left|U_{4}\right\rangle\right), & \left|\Sigma_{4}\right\rangle=\frac{1}{\sqrt{2}}\left(\left|U_{3}\right\rangle+\left|U_{4}\right\rangle\right) .
\end{array}
$$

Now we introduce four vectors $\left|D_{\alpha}\right\rangle ; \alpha=1,2,3,4$ of the form

$$
\left|D_{1}\right\rangle=\left(\begin{array}{l}
0 \\
1 \\
1 \\
0
\end{array}\right), \quad\left|D_{2}\right\rangle=\left(\begin{array}{c}
0 \\
-i \\
i \\
0
\end{array}\right), \quad\left|D_{3}\right\rangle=\left(\begin{array}{c}
1 \\
(-1+i) / 2 \\
(-1-i) / 2 \\
0
\end{array}\right), \quad\left|D_{4}\right\rangle=\left(\begin{array}{c}
0 \\
(-1+i) / 2 \\
(-1-i) / 2 \\
1
\end{array}\right)
$$

These vectors determine four Hermitian matrices $D_{\alpha}$,

$$
\begin{aligned}
D_{1} & =\left(\begin{array}{ll}
0 & 1 \\
1 & 0
\end{array}\right), & D_{2} & =\left(\begin{array}{cc}
0 & -i \\
i & 0
\end{array}\right) \\
D_{3} & =\left(\begin{array}{cc}
1 & (-1+i) / 2 \\
(-1-i) / 2 & 0
\end{array}\right), & D_{4} & =\left(\begin{array}{cc}
0 & (-1+i) / 2 \\
(-1-i) / 2 & 1
\end{array}\right) .
\end{aligned}
$$


One can check that vectors $\left|D_{j}\right\rangle$ and $\left|U_{k}\right\rangle$ satisfy the condition

$$
\left\langle D_{j} \mid U_{k}\right\rangle=\delta_{j k}
$$

this condition provides the possibility to represent any four-vector $|A\rangle$

$$
\text { either as } \quad|A\rangle=\sum_{j=1}^{4}\left\langle D_{j} \mid A\right\rangle\left|U_{j}\right\rangle \quad \text { or as } \quad|A\rangle=\sum_{j=1}^{4}\left\langle U_{j} \mid A\right\rangle\left|D_{j}\right\rangle \text {. }
$$

The matrix $A$ corresponding to the vector $|A\rangle$, in view of the above rule, can be written as follows:

$$
A=\sum_{j=1}^{4}\left[\operatorname{Tr}\left(A D_{j}\right)\right] U_{j}, \quad A=\sum_{j=1}^{4}\left[\operatorname{Tr}\left(A U_{j}\right)\right] D_{j} .
$$

Following [22], we call matrices $U_{j}$ the dequantizers and matrices $D_{j}$, the quantizers.

\section{The von Neumann Evolution Equation for the Density Matrix $\rho$ in Vector Form}

The density matrix $\rho(t)$ of the system with Hamiltonian $H$ obeys the von Neumann evolution equation [5]

$$
\frac{\partial \rho(t)}{\partial t}+i(H \rho(t)-\rho(t) H)=0 .
$$

This linear matrix equation can be written in the following form of the system of equations for the matrix elements $\rho_{j k}(t)$ of the density matrix $\rho(t)$ of the spin- $1 / 2$ particle:

$$
\begin{aligned}
\frac{\partial \rho_{11}(t)}{\partial t}+i\left[H_{12} \rho_{21}(t)-H_{21} \rho_{12}(t)\right] & =0, \\
\frac{\partial \rho_{12}(t)}{\partial t}+i\left[\left(H_{11}-H_{22}\right) \rho_{12}(t)-H_{12}\left(\rho_{22}(t)-\rho_{11}(t)\right)\right] & =0, \\
\frac{\partial \rho_{21}(t)}{\partial t}+i\left[\left(H_{21}\left(\rho_{11}(t)-\rho_{22}(t)\right)+\left(H_{22}-H_{11}\right) \rho_{21}(t)\right]\right. & =0, \\
\frac{\partial \rho_{22}(t)}{\partial t}+i\left[H_{21} \rho_{12}(t)-H_{12} \rho_{21}(t)\right] & =0 .
\end{aligned}
$$

The system of these equations can be rewritten in the form of equation for the vector $|R(t)\rangle$ as follows:

$$
\frac{d}{d t}|R(t)\rangle=M|R(t)\rangle
$$

where the matrix $M$ expressed in terms of Hamiltonian matrix elements reads

$$
M=\left(\begin{array}{cccc}
0 & i H_{21} & -i H_{12} & 0 \\
i H_{12} & -i\left(H_{22}-H_{11}\right) & 0 & -i H_{12} \\
-i H_{21} & 0 & -i\left(H_{11}-H_{22}\right) & i H_{21} \\
0 & -i H_{21} & i H_{12} & 0
\end{array}\right)
$$

The matrix $M$ has the properties $\operatorname{Tr} M=0, M^{+}=-M$, and the eigenvalues of matrix $M$ are imaginary numbers, i.e., $\operatorname{Re} M=0$. The matrix $(i M)=\mathcal{H}$ is Hermitian matrix (an analog of a Hamiltonian), and its diagonal elements are real numbers. If the Hamiltonian $\mathcal{H}$ does not depend on time, the solution of Equation (40) for the vector $|R(t)\rangle$ reads

$$
|R(t)\rangle=\exp (M t)|R(0)\rangle=e^{-i \mathcal{H} t}|R(0)\rangle .
$$

Taking into account the property of matrix $M$, we state that the $4 \times 4$-matrix $\exp (M t)$ is unitary matrix. 
We will show that Equation (40) can be presented in the form of linear kinetic equation for probability distributions of classical-like random variables.

\section{The Probability Representation of the von Neumann Equation}

We transform the von Neumann Equation (38) written for the complex vector $|R(t)\rangle$ to the kinetic equation written for the probability vector $|P(t)\rangle=\left(\begin{array}{l}p_{1}(t) \\ p_{2}(t) \\ p_{3}(t) \\ p_{4}(t)\end{array}\right)$, where the functions of time $0 \leq p_{1}(t), p_{2}(t), p_{3}(t) \leq 1$ are the probabilities of spin- $1 / 2$ projections $m=+1 / 2$ onto $x, y$, and $z$ directions at any moment of time $t$. Probability $p_{4}(t)=1-p_{3}(t)$ is the probability of spin projection $m=-1 / 2$ on the $z$ direction.

Using Equations (39)-(42), we introduce the linear invertible transform of vectors $|R(t)\rangle$ which maps this vector onto the probability vector $|P(t)\rangle$ of the form

$$
|P(t)\rangle=T|R(t)\rangle+|\Gamma\rangle .
$$

One can check that the $4 \times 4$-matrix $T$ and the 4 -vector $\Gamma$ are

$$
T=\left(\begin{array}{cccc}
0 & 1 / 2 & 1 / 2 & 0 \\
0 & i / 2 & -i / 2 & 0 \\
1 & 0 & 0 & 0 \\
0 & 0 & 0 & 1
\end{array}\right), \quad|\Gamma\rangle=\left(\begin{array}{c}
1 / 2 \\
1 / 2 \\
0 \\
0
\end{array}\right)
$$

The inverse transform

$$
|R(t)\rangle=T^{-1}(|P(t)\rangle-|\Gamma\rangle), \quad T^{-1}=\left(\begin{array}{cccc}
0 & 0 & 1 & 0 \\
1 & -i & 0 & 0 \\
1 & i & 0 & 0 \\
0 & 0 & 0 & 1
\end{array}\right)
$$

relates the matrix elements $\rho_{j k}$ with probabilities given by (23).

Equation (43) means that

$$
\frac{d}{d t}|P(t)\rangle=T \frac{d}{d t}|R(t)\rangle
$$

In view of (40) and (45), we obtain the kinetic equation

$$
\frac{d}{d t}|P(t)\rangle=\left(T M T^{-1}\right)|P(t)\rangle-\left(T M T^{-1}\right)|\Gamma\rangle .
$$

Since the $4 \times 4$-matrix $M$ is given by (41) and is equal to $-i \mathcal{H}$, where the matrix $\mathcal{H}$ is the Hermitian matrix analogous to the Hamiltonian describing the evolution of an artificial ququart, the matrix $T M T^{-1}$ is expressed in terms of the Hamiltonian matrix elements of the spin- $1 / 2$ system $H_{j k}$ as we show in the following.

Equation (39) can be written for a simplest example of the diagonal stationary Hamiltonian, where $H_{12}=H_{21}=0, H_{12}=E_{1}$, and $H_{22}=E_{2}$. In this case, the matrix $M$ has only two nonzero matrix elements $M_{22}=-M_{33}=-i\left(E_{2}-E_{1}\right)=i \omega$. These equations mean that

$$
\dot{\rho}_{11}(t)=\dot{\rho}_{22}(t)=0, \quad \dot{\rho}_{12}(t)=i \omega \rho_{12}(t), \quad \dot{\rho}_{21}(t)=-i \omega \rho_{21}(t)
$$

Solutions to these equations provide the constant probability of spin projection $m=+1 / 2$ on the $z$ axis, i.e., $\rho_{11}(t)=p_{3}$ and $\rho_{22}(t)=1-p_{3}$. The off-diagonal matrix elements of the density matrix $\rho_{12}(t)=\rho_{21}^{*}(t)$ are 


$$
\rho_{12}(t)=e^{i \omega t} \rho_{12}(0) ;
$$

that provides the expression for the probabilities $p_{1}(t)$ and $p_{2}(t)$ of the form

$$
\left[p_{1}(t)-1 / 2\right]-i\left[p_{2}(t)-1 / 2\right]=[\cos \omega t+i \sin \omega t]\left[\left(p_{1}-1 / 2\right)-i\left(p_{2}-1 / 2\right)\right] .
$$

The probabilities $p_{1}(t), p_{2}(t)$, and $p_{3}(t)$ read

$$
\begin{aligned}
& p_{1}(t)=(1 / 2)+\cos \omega t\left(p_{1}-1 / 2\right)+\sin \omega t\left(p_{2}-1 / 2\right), \\
& p_{2}(t)=(1 / 2)-\sin \omega t\left(p_{1}-1 / 2\right)+\cos \omega t\left(p_{2}-1 / 2\right), \\
& p_{3}(t)=p_{3} ; \quad p_{1}=p_{1}(t=0) ; \quad p_{2}=p_{2}(t=0) ; \quad p_{3}=p_{3}(t=0) .
\end{aligned}
$$

The probabilities satisfy the condition

$$
\sum_{j=1}^{3}\left[p_{j}(t)-1 / 2\right]^{2}=\sum_{j=1}^{3}\left[p_{j}-1 / 2\right]^{2}
$$

The probabilities $p_{1}, p_{2}$, and $p_{3}$, determining the density matrix $\rho$ given in the initial basis $|1 / 2\rangle,|-1 / 2\rangle$, are changed to be converted to the probabilities $\tilde{p}_{1}, \tilde{p}_{2}$, and $\tilde{p}_{3}$ related to the density matrix $\rho_{u}$, corresponding to the basis obtained using the unitary transform of the initial basis given by the matrix $u$, such that $u u^{\dagger}=1$ and $u \rho u^{\dagger}=\rho_{u}$. The explicit expressions for the probabilities $\tilde{p}_{1}, \tilde{p}_{2}$, and $\tilde{p}_{3}$ read

$$
\begin{aligned}
\tilde{p}_{1}= & (1 / 2)+p_{1}\left[\operatorname{Re}\left(u_{22} u_{11}^{*}+u_{21} u_{12}^{*}\right)+\operatorname{Re}\left(u_{22} u_{11}^{*}\right)\right] \\
& +p_{2}\left[-\operatorname{Im}\left(u_{22} u_{11}^{*}\right)+\operatorname{Im}\left(u_{21} u_{12}^{*}\right)\right]+p_{3}\left[\operatorname{Re}\left(u_{21} u_{11}^{*}-u_{22} u_{12}^{*}\right)\right] \\
& -(1 / 2)\left[\operatorname{Re}\left(u_{22} u_{11}^{*}\right)-\operatorname{Im}\left(u_{22} u_{11}^{*}\right)+\operatorname{Re}\left(u_{21} u_{12}^{*}\right)+\operatorname{Im}\left(u_{21} u_{12}^{*}\right)\right]+\operatorname{Re}\left(u_{22} u_{12}^{*}\right), \\
\tilde{p}_{2}= & (1 / 2)+p_{1}\left[\operatorname{Im}\left(u_{22} u_{11}^{*}+u_{21} u_{12}^{*}\right)\right]+p_{2}\left[\operatorname{Re}\left(u_{22} u_{11}^{*}-u_{21} u_{12}^{*}\right)\right] \\
& +p_{3}\left[\operatorname{Im}\left(u_{21} u_{11}^{*}-u_{22} u_{12}^{*}\right)\right]-(1 / 2)\left[\operatorname{Im}\left(u_{22} u_{11}^{*}\right)+\operatorname{Re}\left(u_{22} u_{11}^{*}\right)\right. \\
& \left.+\operatorname{Im}\left(u_{21} u_{12}^{*}\right)-\operatorname{Re}\left(u_{21} u_{12}^{*}\right)\right]+\operatorname{Im}\left(u_{22} u_{12}^{*}\right), \\
\tilde{p}_{3}= & 2 p_{1}\left[\operatorname{Re}\left(u_{11}^{*} u_{12}\right)\right]+2 p_{2}\left[\operatorname{Im}\left(u_{11} u_{12}^{*}\right)\right]+p_{3}\left[\left|u_{11}\right|^{2}-\left|u_{12}\right|^{2}\right] \\
& +\left\{\left|u_{12}\right|^{2}-\left[\operatorname{Re}\left(u_{11} u_{12}^{*}\right)\right]+\left[\operatorname{Im}\left(u_{11}^{*} u_{12}\right)\right]\right\} .
\end{aligned}
$$

We can use formulas (53)-(55) to relate the probabilities $p_{1}(t), p_{2}(t)$, and $p_{3}(t)$ given by (51) to the probabilities associated with the solution to the kinetic equation determined by generic Hermitian Hamiltonian matrix $H_{j k} ; j, k=1,2$. For this, we express the matrix elements of the unitary matrix $u_{j k}$; $j, k=1,2$ through the matrix elements of the Hamiltonian satisfying the matrix condition

$$
H u=u H_{E}, \quad H_{E}=\left(\begin{array}{cc}
E_{1} & 0 \\
0 & E_{2}
\end{array}\right)
$$

where the eigenvalues of the Hamiltonian $H$ are

$$
E_{1,2}=\frac{1}{2}\left\{\left(H_{11}+H_{22}\right) \pm\left[\left(H_{11}+H_{22}\right)^{2}-4 H_{11} H_{22}+4\left|H_{12}\right|^{2}\right]^{1 / 2}\right\} .
$$


The matrix elements of the unitary matrix $u$ are expressed in terms of the matrix elements of the Hamiltonian as follows:

$$
\begin{aligned}
& u_{11}=\left\{1+\left|H_{12}^{-1}\left[\frac{H_{22}-H_{11}}{2}+\left(\frac{\left(H_{11}+H_{22}\right)^{2}}{4}-\left(H_{11} H_{22}-\left|H_{12}\right|^{2}\right)\right)^{1 / 2}\right]\right|^{2}\right\}^{-1 / 2}, \\
& u_{21}=u_{11} H_{12}^{-1}\left[\frac{H_{22}-H_{11}}{2}+\left(\frac{\left(H_{11}+H_{22}\right)^{2}}{4}-\left(H_{11} H_{22}-\left|H_{12}\right|^{2}\right)\right)^{1 / 2}\right], \\
& u_{22}=\left\{1+\left|H_{21}^{-1}\left[\frac{H_{11}-H_{22}}{2}-\left(\frac{\left(H_{11}+H_{22}\right)^{2}}{4}-\left(H_{11} H_{22}-\left|H_{12}\right|^{2}\right)\right)^{1 / 2}\right]\right|^{2}\right\}^{-1 / 2}, \\
& u_{12}=u_{22} H_{21}^{-1}\left[\frac{H_{11}-H_{22}}{2}-\left(\frac{\left(H_{11}+H_{22}\right)^{2}}{4}-\left(H_{11} H_{22}-\left|H_{12}\right|^{2}\right)\right)^{1 / 2}\right] .
\end{aligned}
$$

Thus, the unitary matrix $u$ is expressed in terms of the Hamiltonian, and the matrix elements given by (58)-(61) satisfy the relations

$$
\left|u_{11}\right|^{2}+\left|u_{21}\right|^{2}=\left|u_{12}\right|^{2}+\left|u_{22}\right|^{2}=1, \quad u_{11}^{*} u_{12}+u_{21}^{*} u_{22}=0 .
$$

Thus, relations (51) considered in the basis, where the Hamiltonian $H$ has the diagonal form providing the evolution of the density matrix, can be transformed by means of the matrix $u$ given by (57)-(61), using the basis where the Hamiltonian has generic form with nonzero off-diagonal matrix elements.

The constructed invertible map of the density matrix onto probabilities $p_{1}, p_{2}$, and $p_{3}$ and of the von Neumann equation on the system of Equation (47) can be interpreted as follows. The solutions to the quantum kinetic equations can be considered as trajectories in a real three-dimensional space satisfying the system of the first-order differential equations. Trajectories of classical mechanical systems with three degrees of freedom, e.g., classical oscillator, satisfy the system of the second-order differential equations.

For qubit state, the trajectory satisfying the system of Equation (47) has the constant value $\left[p_{1}(t)-1 / 2\right]^{2}+\left[p_{2}(t)-1 / 2\right]^{2}+\left[p_{3}(t)-1 / 2\right]^{2}$, which means the invariance of the purity parameter of quantum states during the time evolution. In the probability representation, the qubit evolution is described by the motion of a point with coordinates $p_{1}(t), p_{2}(t)$, and $p_{3}(t)$ on the surface of the sphere with radius determined by the purity parameter of the state, which is a function of the probabilities.

Now we consider all four-vectors $\left|\rho^{(\beta)}\right\rangle$, which can be expressed as linear combinations (convex sum) of vectors (29),

$$
\left|\rho^{(\beta)}\right\rangle=\sum_{\alpha=1}^{4} \mathcal{P}_{\alpha}^{(\beta)}\left|U_{\alpha}\right\rangle
$$

where $0 \leq \mathcal{P}_{\alpha}^{(\beta)} \leq 1, \sum_{\alpha=1}^{4} \mathcal{P}_{\alpha}^{(\beta)}=1$, and the set of indices $\beta$ can be either finite or infinite one. In view of Born's rule, the following condition is valid

$$
\left\langle\rho^{\left(\beta_{1}\right)} \mid \rho^{\left(\beta_{2}\right)}\right\rangle=\Pi_{\beta_{1}}^{\beta_{2}} .
$$

Here, we used the invertible map (21) of the density matrices onto the vectors and calculated scalar products of vectors (64), which can be interpreted as probabilities satisfying the condition $0 \leq \Pi_{\beta_{1}}^{\beta_{2}} \leq 1$.

Thus, in the four-dimensional Hilbert space of arbitrary vectors $|H\rangle=\sum_{\alpha=1}^{4} H_{\alpha}\left|U_{\alpha}\right\rangle$, where $H_{\alpha}$ are complex numbers, there exists the subset (convex sum of vectors $\left|U_{\alpha}\right\rangle$ ) corresponding to the set 
of density $4 \times 4$-matrices of physical-system states. We will address the problem of symmetry of this subset and its relation to the $O(3)$ symmetry group associated with two-dimensional irreducible representation of the unitary group $S U(2)$.

The $S U(2)$ symmetry plays in our consideration an important role since the Hamiltonian $H$ is connected with the $s u(2)$ Lie algebra, and the matrix $u$ and $\exp (-i H t)$ are matrices of irreducible representation of the unitary group.

\section{7. $O(3)$ Transforms of Probabilities and Spin-Projection Mean Values}

In this section, we consider the spin-projection mean-value representation of qubit states. This representation uses Bloch parameters determining the density matrix of the form

$$
\hat{\rho}=\frac{1}{2}\left(\begin{array}{cc}
1+z & x-i y \\
x+i y & 1-z
\end{array}\right), \quad x^{2}+y^{2}+z^{2} \leq 1,
$$

where $x, y$, and $z$ are mean values of spin projections on the $\vec{X}, \vec{Y}$, and $\vec{Z}$ axes, respectively. The probabilities $p_{1}, p_{2}$, and $p_{3}$ are the result of measuring the spin projections in state (65) on the axes given by the basis vectors $\vec{X}, \vec{Y}$, and $\vec{Z}$; they are nonnegative and are related to the mean values $x, y$, and $z$ as follows:

$$
p_{1}=(1+x) / 2, \quad p_{2}=(1+y) / 2, \quad p_{3}=(1+z) / 2 .
$$

In the case of one-qubit state, the relationship between the probabilities and mean values is very simple, but for $\mathrm{N}$-qubit states, it is more complicated; for details, see [52-55].

In the state space described by the density matrix (65), one can define the action of the $S U(2)$ group,

$$
\tilde{\rho}=u \hat{\rho} u^{\dagger}=\left(\begin{array}{cc}
\tilde{\rho}_{11} & \tilde{\rho}_{12} \\
\tilde{\rho}_{21} & \tilde{\rho}_{22}
\end{array}\right) .
$$

The unitary transform $u \in S U(2)$ is defined by the matrix parameterized by Euler angles,

$$
u=\left(\begin{array}{cc}
(\cos \vartheta / 2) e^{i(\varphi+\psi) / 2} & (\sin \vartheta / 2) e^{i(\varphi-\psi) / 2} \\
-(\sin \vartheta / 2) e^{i(-\varphi+\psi) / 2} & (\cos \vartheta / 2) e^{-i(\varphi+\psi) / 2}
\end{array}\right)
$$

The matrix elements of the density matrix $\tilde{\rho}$ read

$$
\begin{aligned}
\tilde{\rho}_{11}= & \frac{1}{2}[1+\cos \vartheta z+\sin \vartheta(\cos \psi x+\sin \psi y)], \\
\tilde{\rho}_{12}= & \frac{1}{2}[\cos \varphi(-\sin \vartheta z+\cos \vartheta(\cos \psi x+\sin \psi y))-\sin \varphi(\sin \psi x-\cos \psi y)] \\
& +\frac{i}{2}[\sin \varphi(-\sin \vartheta z+\cos \vartheta(\cos \psi x-\sin \psi y))+\cos \varphi(\sin \psi x-\cos \psi y)], \\
\tilde{\rho}_{21}= & \tilde{\rho}_{12}^{*}, \quad \tilde{\rho}_{22}=1-\tilde{\rho}_{11} .
\end{aligned}
$$

The transformed density matrix $\tilde{\rho}$ can be written as

$$
\tilde{\hat{\rho}}=\frac{1}{2}\left(\begin{array}{cc}
1+\tilde{z} & \tilde{x}-i \tilde{y} \\
\tilde{x}+i \tilde{y} & 1-\tilde{z}
\end{array}\right),
$$


where the values $\tilde{x}, \tilde{y}$, and $z$ have the sense of mean values of the spin projections on the $\vec{X}, \vec{Y}$, and $\vec{Z}$ axes in the case where the state is described by the density matrix (67); these mean values read

$$
\begin{aligned}
& \tilde{x}=(\cos \varphi \cos \vartheta \cos \psi-\sin \varphi \sin \psi) x-(\cos \varphi \cos \vartheta \sin \psi+\sin \varphi \cos \psi) y-\cos \varphi \sin \vartheta z, \\
& \tilde{y}=(\cos \varphi \sin \psi+\sin \varphi \cos \vartheta \cos \psi) x+(-\sin \varphi \cos \vartheta \sin \psi+\cos \varphi \cos \psi) y-\sin \varphi \sin \vartheta z, \\
& \tilde{z}=\sin \vartheta \cos \psi x-\sin \vartheta \sin \psi y+\cos \vartheta z .
\end{aligned}
$$

The spin-projection mean values $x, y$, and $z$ can be mapped onto three vectors in the three-dimensional configuration space $E(3)$,

$$
\vec{x}=x\left(\begin{array}{l}
1 \\
0 \\
0
\end{array}\right), \quad \vec{y}=y\left(\begin{array}{l}
0 \\
1 \\
0
\end{array}\right), \quad \vec{z}=z\left(\begin{array}{l}
0 \\
0 \\
1
\end{array}\right) ;
$$

these orthogonal vectors form the basis. Similar vectors can be used for describing the transformed values $\tilde{x}, \tilde{y}$, and $\tilde{z}$. Then the unitary transform (67) generates an orthogonal transform in the vector space

$$
\left(\begin{array}{l}
\tilde{x} \\
\tilde{y} \\
\tilde{z}
\end{array}\right)=\mathcal{O}\left(\begin{array}{l}
x \\
y \\
z
\end{array}\right)=\left(\begin{array}{lll}
O_{11} & O_{12} & O_{13} \\
O_{21} & O_{22} & O_{33} \\
O_{31} & O_{32} & O_{33}
\end{array}\right)\left(\begin{array}{l}
x \\
y \\
z
\end{array}\right)
$$

with the orthogonal matrix $\mathcal{O}$ of the form

$$
\mathcal{O}=\left(\begin{array}{ccc}
\cos \varphi \cos \vartheta \cos \psi-\sin \varphi \sin \psi & -\cos \varphi \cos \vartheta \sin \psi-\sin \varphi \cos \psi & -\cos \varphi \sin \vartheta \\
\cos \varphi \sin \psi+\sin \varphi \cos \vartheta \cos \psi) & -\sin \varphi \cos \vartheta \sin \psi+\cos \varphi \cos \psi & -\sin \varphi \sin \vartheta \\
\sin \vartheta \cos \psi & -\sin \vartheta \sin \psi & \cos \vartheta
\end{array}\right) .
$$

The orthogonal $3 \times 3$-matrix $\mathcal{O}$ transforms the three-vector $\vec{r}=(x, y, z)$ to the three-vector $\vec{r}^{\prime}=(\tilde{x}, \tilde{y}, \tilde{z})$. Thus, the unitary transform (67) of the density matrix generates an orthogonal transform (73) of the mean values $x, y$, and $z$.

Now we clarify how the probabilities $p_{1}, p_{2}$, and $p_{3}$ are transformed; for this, we apply relations (66), namely, after substituting (66) into (73), we arrive at the following equations:

$$
\begin{aligned}
& \tilde{p}_{1}=O_{11} p_{1}+O_{12} p_{2}+O_{13} p_{3}+\left(1-O_{11}-O_{12}-O_{13}\right) / 2, \\
& \tilde{p}_{2}=O_{21} p_{1}+O_{22} p_{2}+O_{23} p_{3}+\left(1-O_{21}-O_{22}-O_{23}\right) / 2, \\
& \tilde{p}_{3}=O_{31} p_{1}+O_{32} p_{2}+O_{33} p_{3}+\left(1-O_{31}-O_{32}-O_{33}\right) / 2 .
\end{aligned}
$$

In matrix form, the system of equations (75) reads

$$
\left(\begin{array}{l}
\tilde{p}_{1} \\
\tilde{p}_{2} \\
\tilde{p}_{3}
\end{array}\right)=\left(\begin{array}{lll}
O_{11} & O_{12} & O_{13} \\
O_{21} & O_{22} & O_{33} \\
O_{31} & O_{32} & O_{33}
\end{array}\right)\left(\begin{array}{l}
p_{1} \\
p_{2} \\
p_{3}
\end{array}\right)+\frac{1}{2}\left(\begin{array}{l}
1-O_{11}-O_{12}-O_{13} \\
1-O_{21}-O_{22}-O_{23} \\
1-O_{31}-O_{32}-O_{33}
\end{array}\right)
$$

Thus, we conclude that the unitary transform (67) of the density matrix generates an orthogonal transform (73) of the spin-projection mean values $x, y$, and $z$ and transform (76), where we applied a nonuniform orthogonal group (rotation $\mathcal{O}$ and shift $\mathcal{T}$ ) of probabilities $p_{1}, p_{2}$, and $p_{3}$. Transform (76) corresponds to relations (53)-(55) written in the form of orthogonal transform of the probabilities $p_{1}$, $p_{2}$, and $p_{3}$. 
As an example, we consider the unitary transform

$$
u=\left(\begin{array}{cc}
\cos \vartheta / 2 & \sin \vartheta / 2 \\
-\sin \vartheta / 2 & \cos \vartheta / 2
\end{array}\right) .
$$

In this case, the transformed density matrix $\tilde{\rho}$ reads

$$
\tilde{\rho}=\frac{1}{2}\left(\begin{array}{cc}
1+\sin \vartheta x+\cos \vartheta z & \cos \vartheta x+i y \\
\cos \vartheta x-i y & 1-\sin \vartheta x-\cos \vartheta z
\end{array}\right)
$$

and the orthogonal matrix $\mathcal{O}$ and the shift vector $\mathcal{T}$ are

$$
\mathcal{O}=\left(\begin{array}{ccc}
\cos \vartheta & 0 & -\sin \vartheta \\
0 & 1 & 0 \\
\sin \vartheta & 0 & \cos \vartheta
\end{array}\right), \quad \mathcal{T}=\left(\begin{array}{c}
(1-\cos \vartheta+\sin \vartheta) / 2 \\
0 \\
(1-\cos \vartheta+\sin \vartheta) / 2
\end{array}\right)
$$

The mean values of the spin projections are transformed as follows:

$$
\tilde{x}=\cos \vartheta x-\sin \vartheta z, \quad \tilde{y}=y, \quad \tilde{z}=\sin \vartheta x+\cos \vartheta z,
$$

and the probabilities are transformed as follows:

$$
\begin{aligned}
& \tilde{p}_{1}=\cos \vartheta p_{1}-\sin \vartheta p_{3}+(1-\cos \vartheta+\sin \vartheta) / 2 \\
& \tilde{p}_{2}=p_{2} \\
& \tilde{p}_{3}=\sin \vartheta p_{1}+\cos \vartheta p_{3}+(1-\cos \vartheta+\sin \vartheta) / 2 .
\end{aligned}
$$

\section{Transforms of Quantizer and Dequantizer Operators}

In this section, we consider the other approach to constructing the spin-projection averages and the projection probabilities; our approach is based on applying the quantizer-dequantizer operators. As we already mentioned, each set of dequantizers maps any operator onto a scalar function called the symbol of this operator.

This function can take both continuous and discrete values, and these values can be used to recover the original operator. As such an operator, we consider the density operator. For a single-qubit state, the density operator has the form (65) and can be represented as a Hermitian $2 \times 2$-matrix. In this case, the minimum set of dequantizers contains four operators, and this set is not unique. We will use the set of dequantizers (29).

Using these dequantizers, we can find nonnegative values of the probabilities of spin projections on the $\vec{X}, \vec{Y}$, and $\vec{Z}$ axes in the state (65); the probabilities read

$$
\begin{aligned}
& p_{1}=\operatorname{Tr} \hat{\rho} \hat{U}^{(1)}=(1+x) / 2, \quad p_{2}=\operatorname{Tr} \hat{\rho} \hat{U}^{(2)}=(1+y) / 2, \\
& p_{3}=\operatorname{Tr} \hat{\rho} \hat{U}^{(3)}=(1+z) / 2, \quad p_{4}=1-p_{3}=\operatorname{Tr} \hat{\rho} \hat{U}^{(4)}=(1-z) / 2 \text {, }
\end{aligned}
$$

and the set of quantizers is given by (34).

The sets of operators presented by matrices (29) and (34) satisfy the orthogonality condition $\operatorname{Tr} \hat{U}^{(i)} \hat{D}^{(j)}=\delta_{i j} ; i, j=1, \ldots, 4$.

The values $p_{1}, p_{2}, p_{3}$, and $p_{4}$ describe the symbol of the density operator (65) constructed using dequantizers (29). They completely define this operator, and it can be reconstructed using the quantizers; the reconstruction formula is $\hat{\rho}=\sum_{k=1}^{4} p_{k} \hat{D}^{(k)}$.

If the probabilities $p_{1}, p_{2}, p_{3}$, and $p_{4}$ are known, one can reconstruct the density matrix of the initial state. The probabilities $p_{1}, p_{2}, p_{3}$, and $p_{4}$ can be obtained as measured probabilities of spin projections onto the $\vec{X}, \vec{Y}$, and $\vec{Z}$ axes. Nevertheless, one can select other axes and measure the 
probabilities of spin projections onto the selected axes. These new probabilities $\tilde{p}_{1}, \tilde{p}_{2}, \tilde{p}_{3}$, and $\tilde{p}_{4}$ correspond to the other set of dequantizers obtained from the initial operators, using the unitary transform $\widetilde{\hat{U}}^{(i)}=u \hat{U}^{(i)} u^{\dagger}, \quad \widetilde{D}^{(j)}=u \hat{D}^{(j)} u^{\dagger} ; i, j=1, \ldots, 4$. It is easy to see that the orthogonality condition holds for these new operators. We obtained the probabilities $\tilde{p}_{1}, \tilde{p}_{2}, \tilde{p}_{3}$, and $\tilde{p}_{4}$, in view of the relations of these numbers with new dequantizers, i.e., $\tilde{p}_{1}=\operatorname{Tr} \hat{\rho} \widetilde{\hat{U}}^{(1)}, \tilde{p}_{2}=\operatorname{Tr} \hat{\rho} \widetilde{\hat{U}}^{(2)}, \tilde{p}_{3}=\operatorname{Tr} \hat{\rho} \widetilde{\hat{U}}^{(3)}$, and $\tilde{p}_{4}=1-\tilde{p}_{3}=\operatorname{Tr} \hat{\rho} \widetilde{\hat{U}}^{(4)}$. Using these probabilities and new quantizers, one can reconstruct the initial density operator $\hat{\rho}=\sum_{k=1}^{4} \tilde{p}_{k} \widetilde{\hat{D}}^{(k)}$.

Thus, we showed that the probabilities of spin projections are determined by the density operator and dequantizers, and these probabilities can be transformed using the unitary transforms of dequantizers with which they are constructed. The similar reasoning can be implemented using other measured values, that is, the average values of spin projections. For this purpose, consider the following set of dequantizers $\hat{U}_{S}^{(1)}=\left(\begin{array}{ll}0 & 1 \\ 1 & 0\end{array}\right), \hat{U}_{S}^{(2)}=\left(\begin{array}{cc}0 & -i \\ i & 0\end{array}\right), \quad \hat{U}_{S}^{(3)}=\left(\begin{array}{cc}1 & 0 \\ 0 & -1\end{array}\right)$, and $\hat{U}_{S}^{(4)}=\left(\begin{array}{ll}1 & 0 \\ 0 & 1\end{array}\right)$. This set of dequantizers provides the set of corresponding quantizers that are $\hat{D}_{S}^{(1)}=\frac{1}{2} \hat{U}_{S}^{(1)}, \hat{D}_{S}^{(2)}=\frac{1}{2} \hat{U}_{S}^{(2)}, \hat{D}_{S}^{(3)}=\frac{1}{2} \hat{U}_{S}^{(3)}$, and $\hat{D}_{S}^{(4)}=\frac{1}{2} \hat{U}_{S}^{(4)}$. These operators satisfy the orthogonality condition $\operatorname{Tr} \hat{U}_{S}^{(i)} \hat{D}_{S}^{(j)}=\delta_{i j} ; \quad i, j=1, \ldots, 4$. The Bloch parameters in the density matrix introduced in (65), in view of dequantizers $\hat{U}_{S}^{(j)} ; j=1,2,3,4$, read $\operatorname{Tr} \hat{\rho} \hat{U}_{S}^{(1)}=s_{1}=x$, $\operatorname{Tr} \hat{\rho} \hat{U}_{S}^{(2)}=s_{2}=y, \operatorname{Tr} \hat{\rho} \hat{U}_{S}^{(3)}=s_{3}=z$, and $\operatorname{Tr} \hat{\rho} \hat{U}_{S}^{(4)}=s_{4}=1$.

One can reconstruct the density matrix (65) using quantizers $\hat{D}_{S}^{(k)}$ and numbers $s_{k}$. The result is

$$
\hat{\rho}=\sum_{k=1}^{4} s_{k} \hat{D}_{S}^{(k)}
$$

As in the previous case, we consider the unitary transform of operators $\hat{U}_{S}^{(j)}$ and $\hat{D}_{S}^{(j)}$

$$
\widetilde{\hat{U}}_{S}^{(i)}=u \hat{U}_{S}^{(i)} u^{\dagger}, \quad \widetilde{\hat{D}}_{S}^{(j)}=u \hat{D}_{S}^{(j)} u^{\dagger} ; \quad i, j=1, \ldots, 4 .
$$

Applying this set of dequantizers, one can obtain new Bloch parameters for the density operator (65); they read

$$
\tilde{s}_{1}=\operatorname{Tr} \hat{\rho} \widetilde{\hat{U}}_{S}^{(1)}, \quad \tilde{s}_{2}=\operatorname{Tr} \hat{\rho} \widetilde{\hat{U}}_{S}^{(2)}, \quad \tilde{s}_{3}=\operatorname{Tr} \hat{\rho} \widetilde{\hat{U}}_{S}^{(3)}, \quad \tilde{s}_{4}=1=\operatorname{Tr} \hat{\rho} \widetilde{\hat{U}}_{S}^{(4)} .
$$

Now we are in the position to reconstruct the initial density operator (65) using the discussed parameters and quantizers as

$$
\hat{\rho}=\sum_{k=1}^{4} \widetilde{s}_{k} \widetilde{\hat{D}}_{S}^{(k)}
$$

Now we recall that the matrix element $\rho_{11}=w_{\rho}(+)$ is the probability that the result of measuring the spin projection in the state (65) onto the $\vec{Z}$ axis is nonnegative, and the matrix element $\rho_{22}=w_{\rho}(-)$ of the density matrix is also nonnegative, while the sum of these probabilities is equal to unity.

In the case of the transformed density matrix (67), these probabilities have the form

$$
\begin{aligned}
& \tilde{\rho}_{11}=w_{\rho}(+)=\frac{1}{2}[1+\cos \vartheta z+\sin \vartheta \cos \psi x+\sin \vartheta \sin \psi y]=\frac{1}{2}[1+(\vec{n}, \vec{\rho})], \\
& \tilde{\rho}_{22}=w_{\rho}(-)=\frac{1}{2}[1-\cos \vartheta z-\sin \vartheta \cos \psi x-\sin \vartheta \sin \psi y]=\frac{1}{2}[1-(\vec{n}, \vec{\rho})],
\end{aligned}
$$


where the Bloch vector $\vec{\rho}=\vec{x}+\vec{y}+\vec{z}$ is given by (72) and corresponds to a state with the density matrix (65), and $\vec{n}$ is a vector with coordinates $(\sin \vartheta \cos \psi, \sin \vartheta \sin \psi, \cos \vartheta)$. Since $\vec{n}$ is a unit vector, $(\vec{n}, \vec{\rho}) \leq 1$. In fact, the probabilities are determined by tomographic-probability distributions of spin-1/2 states introduced in [16,17]; the probabilities (87) take values in the interval $[(1-|\vec{\rho}|) / 2,(1+|\vec{\rho}|) / 2]$.

For pure states, the length of the Bloch vector $|\vec{\rho}|=1$ and probabilities $w_{\rho}(+)$ and $w_{\rho}(-)$ can take values in the interval $[0,1]$, depending on the direction of $\vec{n}$. For mixed states, the interval of acceptable values is smaller and, in the limit case, it is converted into the point $[1 / 2,1 / 2]$. Such probabilities $w_{\rho}(+)=w_{\rho}(-)=1 / 2$, regardless of the $\vec{n}$ direction, correspond to the mixed state with the density matrix $\hat{\rho}=\frac{1}{2}\left(\begin{array}{ll}1 & 0 \\ 0 & 1\end{array}\right)$. The length of its Bloch vector is equal to zero.

The state (65) is determined by three numbers; as such numbers, one can take three probabilities of the spin projections on three axes. These axes can be selected arbitrarily, the only requirement is that they are not located on the same plane. We fix three probabilities $w_{1}(+), w_{2}(+)$, and $w_{3}(+)$ and write the following three relations:

$$
\begin{aligned}
& w_{1}(+)=\left[1+\cos \vartheta_{1} z+\sin \vartheta_{1} \cos \psi_{1} x+\sin \vartheta_{1} \sin \psi_{1} y\right] / 2, \\
& w_{2}(+)=\left[1+\cos \vartheta_{2} z+\sin \vartheta_{2} \cos \psi_{2} x+\sin \vartheta_{2} \sin \psi_{2} y\right] / 2, \\
& w_{3}(+)=\left[1+\cos \vartheta_{3} z+\sin \vartheta_{3} \cos \psi_{3} x+\sin \vartheta_{3} \sin \psi_{3} y\right] / 2 .
\end{aligned}
$$

Here, angles $\left(\vartheta_{1}, \psi_{1}\right),\left(\vartheta_{2}, \phi_{2}\right)$, and $\left(\vartheta_{3}, \phi_{3}\right)$ determine directions of the reference-frame axes, and $w_{1}(+), w_{2}(+)$, and $w_{3}(+)$ are probabilities of spin projections onto these axes. Relations (88) can be considered as a system of three equations for three unknown quantities, which are Bloch parameters $x, y$, and $z$. The solution of this system of equations provides the density matrix (65).

Now we move to the consideration of the product of three probabilities given by (88),

$$
W_{123}(+++)=w_{1}(+) w_{2}(+) w_{3}(+) .
$$

The product can be understood as the joint probability of spin projections onto the three axes, namely, $\vec{n}_{1} \propto\left(\vartheta_{1}, \psi_{1}\right), \vec{n}_{2} \propto\left(\vartheta_{2}, \psi_{2}\right)$, and $\vec{n}_{3} \propto\left(\vartheta_{3}, \psi_{3}\right)$. Probabilities (88) are independent but they satisfy the nonnegativity condition of the density matrix. One can derive other probabilities:

$$
\begin{aligned}
W_{12}(++)= & \frac{1}{4 \pi} \sum_{m=+}^{-} \int_{0}^{\pi} \int_{0}^{2 \pi} W_{123}(++m)\left(\vartheta_{3}, \psi_{3}\right) \sin \vartheta_{3} d \vartheta_{3} d \psi_{3}=w_{1}(+) w_{2}(+), \\
w_{1}(+)= & \frac{1}{(4 \pi)^{2}} \sum_{m, n=+}^{-} \int_{0}^{\pi} \int_{0}^{\pi} \int_{0}^{2 \pi} \int_{0}^{2 \pi} W_{123}(+n m)\left(\vartheta_{2}, \psi_{2}, \vartheta_{3}, \psi_{3}\right) \\
& \times \sin \vartheta_{2} \sin \vartheta_{3} d \vartheta_{2} d \vartheta_{3} d \psi_{2} d \psi_{3} .
\end{aligned}
$$

We showed that there are two approaches to describe the probabilities determining the quantum state. One of them is based on using the properties of the initial density matrix and its unitary transforms. The other approach applies the interpretation of these probabilities as the symbol of density operator constructed using a certain set of dequantizers and unitary transforms of these operators. In the simplest case of one-qubit state, these approaches are equivalent. For multiqubit states, the problem of their probabilistic description and the symmetries of these discrete characteristics requires additional study. 


\section{Symplectic Tomographic Probability Distribution}

Let us consider the example of the probability representation of quantum states with continuous variables like oscillator states. Using real variables $x=(X, \mu, v)$ where $-\infty<X, \mu, v<\infty$, the dequantizer $\hat{U}(x) \equiv \hat{U}(X, \mu, v)$ is given as

$$
\hat{U}(X, \mu, v)=\delta(X-\mu \hat{q}-v \hat{p}),
$$

with $\hat{q}$ being the position operator and $\hat{p}$, the momentum operator. Below we assume Planck's constant $\hbar=1$.

The quantizer operator $\hat{D}(x) \equiv \hat{D}(X, \mu, v)$ is given as

$$
\hat{D}(X, \mu, v)=\frac{1}{2 \pi} \exp [i(X-\mu \hat{q}-v \hat{p})] .
$$

In the position representation, the matrix elements of dequantizer (92) are

$$
\left\langle y^{\prime}|\delta(X-\mu \hat{q}-v \hat{p})| y\right\rangle=\frac{1}{2 \pi|v|} \exp \left[\frac{i \mu}{2 v}\left(y^{2}-y^{\prime 2}\right)-\frac{i X}{v}\left(y-y^{\prime}\right)\right] .
$$

The quantizer operator (93) has the matrix elements of the form

$$
\left\langle y\left|(2 \pi)^{-1} \exp [i(X-\mu \hat{q}-v \hat{p})]\right| y^{\prime}\right\rangle=\frac{1}{2 \pi} \exp \left(i X-\frac{i \mu \nu}{2}-i \mu y^{\prime}\right) \delta\left(y-y^{\prime}-v\right) .
$$

For the pure state $\hat{\rho}_{\psi}=|\psi\rangle\langle\psi|$, the symbol of operator $\hat{\rho}_{\psi}$ can be evaluated using (94); it reads

$$
w_{\psi}(X \mid \mu, v)=\frac{1}{2 \pi|v|}\left|\int \psi(y) \exp \left(\frac{i \mu y^{2}}{2 v}-\frac{i X y}{v}\right) d y\right|^{2} .
$$

For the wave function such that $\int|\psi(y)|^{2} d y=1$, one has the property

$$
\int w_{\psi}(X \mid \mu, v) d X=1
$$

Formula (96) also provides the nonnegativity of the symbol of operator $\hat{\rho}_{\psi}$, i.e., $w_{\psi}(X \mid \mu, v) \geq 0$.

For an arbitrary density operator which is a convex sum of the pure-state density operators, one has

$$
w_{\rho}(X \mid \mu, v)=\operatorname{Tr}[\hat{\rho} \delta(X-\mu \hat{q}-v \hat{p})] \geq 0,
$$

and the symbol of density operator can be interpreted as the conditional probability distribution of random variable $X$ called the symplectic tomogram.

The symmetry group which we use to construct the symplectic tomographic probability distributions is the Heisenberg-Weyl nilpotent group of real $3 \times 3$-matrices

$$
g(X, \mu, v)=\left(\begin{array}{ccc}
1 & \mu & X \\
0 & 1 & v \\
0 & 0 & 1
\end{array}\right), \quad-\infty<X, \mu, v<\infty ;
$$

Lie algebra of the group is realized by operators $\hat{q}, \hat{p}$ and $\hat{1}$. It is known that operators (93) provide the irreducible unitary ray representation of this group, i.e., the product of the group elements

$$
g\left(X_{1}, \mu_{1}, v_{1}\right) g\left(X_{2}, \mu_{2}, v_{2}\right)=g\left(X_{1}+X_{2}+\mu_{1} v_{2}, \mu_{1}+\mu_{2}, v_{1}+v_{2}\right)
$$


provides the product of operators $2 \pi \hat{D}\left(X_{1}, \mu_{1}, v_{1}\right)$ (93) of the form

$$
\begin{aligned}
& \exp i\left(X_{1}-\mu_{1} \hat{q}-v_{1} \hat{p}\right) \exp i\left(X_{2}-\mu_{2} \hat{q}-v_{2} \hat{p}\right)=\exp \left[i\left(X_{1}+X_{2}\right)+\left(\mu_{1}+\mu_{2}\right) \hat{q}+\left(v_{1}+v_{2}\right) \hat{p}\right] \\
& \times \exp \left[(i / 2)\left(\mu_{1} v_{2}-\mu_{2} v_{1}\right)\right] .
\end{aligned}
$$

The structure constants of the associative product of symbols of operators given by general relation (1) with dequantizer (92) read

$$
K\left(X_{1}, \mu_{1}, v_{1}, X_{2}, \mu_{2}, v_{2}, X, \mu, v\right)=\operatorname{Tr}\left(\hat{D}\left(X_{1}, \mu_{1}, v_{1}\right) \hat{D}\left(X_{2}, \mu_{2}, v_{2}\right) \hat{U}(X, \mu, v)\right),
$$

and we arrive at [22]

$$
\begin{aligned}
& K\left(X_{1}, \mu_{1}, v_{1}, X_{2}, \mu_{2}, v_{2}, X, \mu, v\right)=\frac{\delta\left(\mu\left(v_{1}+v_{2}\right)-v\left(\mu_{1}+\mu_{2}\right)\right)}{4 \pi^{2}} \exp \left(\frac { i } { 2 } \left\{\left(v_{1} \mu_{2}-v_{2} \mu_{1}\right)+2 X_{1}+2 X_{2}\right.\right. \\
& \left.\left.-\left[\left(1-\sqrt{1-4 \mu^{2} v^{2}}\right) \frac{v_{1}+v_{2}}{v}+\left(1+\sqrt{1-4 \mu^{2} v^{2}}\right) \frac{\mu_{1}+\mu_{2}}{\mu}\right] X\right\}\right) .
\end{aligned}
$$

In view of properties of Dirac delta-function, one can express the kernel as

$$
\begin{array}{r}
K\left(X_{1}, \mu_{1}, v_{1}, X_{2}, \mu_{2}, v_{2}, X, \mu, v\right)=\frac{\delta\left(\mu\left(v_{1}+v_{2}\right)-v\left(\mu_{1}+\mu_{2}\right)\right)}{4 \pi^{2}} \\
\quad \times \exp \left(\frac{i}{2}\left\{\left(v_{1} \mu_{2}-v_{2} \mu_{1}\right)+2 X_{1}+2 X_{2}-\frac{2\left(v_{1}+v_{2}\right)}{v} X\right\}\right) .
\end{array}
$$

The corresponding Lie algebra structure constants are

$$
C\left(X_{1}, \mu_{1}, v_{1}, X_{2}, \mu_{2}, v_{2}, X, \mu, v\right)=K\left(X_{1}, \mu_{1}, v_{1}, X_{2}, \mu_{2}, v_{2}, X, \mu, v\right)-K\left(X_{2}, \mu_{2}, v_{2}, X_{1}, \mu_{1}, v_{1}, X, \mu, v\right) ;
$$

in explicit form they read

$$
\begin{aligned}
C\left(X_{1}, \mu_{1}, v_{1}, X_{2}, \mu_{2}, v_{2}, X, \mu, v\right)= & \frac{i}{2 \pi^{2}} \delta\left[\mu\left(v_{1}+v_{2}\right)-v\left(\mu_{1}+\mu_{2}\right)\right] \sin \left(\frac{v_{1} \mu_{2}-v_{2} \mu_{1}}{2}\right) \\
& \times \exp \left(i\left\{X_{1}+X_{2}-\frac{v_{1}+v_{2}}{v} X\right\}\right) .
\end{aligned}
$$

The Lie algebra determined by the structure constants (106) and its connection with the probability representation of quantum system states were not known in the literature.

The structure constants of Jordan product determined by the kernel (102) are

$$
J\left(X_{1}, \mu_{1}, v_{1}, X_{2}, \mu_{2}, v_{2}, X, \mu, v\right)=K\left(X_{1}, \mu_{1}, v_{1}, X_{2}, \mu_{2}, v_{2}, X, \mu, v\right)+K\left(X_{2}, \mu_{2}, v_{2}, X_{1}, \mu_{1}, v_{1}, X, \mu, v\right)
$$

in explicit form they read

$$
\begin{aligned}
J\left(X_{1}, \mu_{1}, v_{1}, X_{2}, \mu_{2}, v_{2}, X, \mu, v\right)= & \frac{1}{2 \pi^{2}} \delta\left[\mu\left(v_{1}+v_{2}\right)-v\left(\mu_{1}+\mu_{2}\right)\right] \cos \left(\frac{v_{1} \mu_{2}-v_{2} \mu_{1}}{2}\right) \\
& \times \exp \left(i\left\{X_{1}+X_{2}-\frac{v_{1}+v_{2}}{v} X\right\}\right) .
\end{aligned}
$$

Thus, for two examples, namely, spin-1/2 systems and systems of continuous variables like, for example, the oscillator, we constructed structure constants of associative products of the symbols of operators and the corresponding Lie algebra structure constants in explicit forms. These examples correspond to the $S U(2)$ symmetry group and the Heisenberg-Weyl nilpotent group.

The new aspects, we obtained for these examples, are their relations with the probability representations of quantum system states. We derived the Lie algebra structure constants, in view of the quantizer-dequantizer operators realizing the description of quantum states by means of probability 
distributions of classical-like random variables. As we showed, the Lie algebras associated to the physical systems also are related to the probabilistic characteristics of the systems, being described by the quantizer-dequantizer operators used to construct the probability picture of quantum system states.

\section{Conclusions}

To conclude, we point out the main results of our work.

We presented the probability representation of qubit states introducing the quantizer-dequantizer operator formalism in an explicit form and calculating the structure constants of the associative product of the symbols of spin operators, as well as the Lie algebra structure constants of the systems associated with $S U(2)$ symmetry.

We obtained in an explicit form the structure constants of the associative algebra and Lie and Jordan algebras using the quantizer-dequantizer operators determining symplectic tomograms of quantum states for systems with continuous degrees of freedom (like oscillator systems). Thus, we found the connection of symmetries (such as Heisenberg-Weyl symmetry) characterized by Lie algebra structures with probability distributions identified with quantum states. The found associative structure constants (104) satisfy generic associativity conditions (13), and Lie algebra structure constants (106) satisfy the Jacobi identity.

The connection of structure constants of Lie algebras with the probability distributions determining the states of quantum systems of both discrete spin variables and continuous oscillator variables is not known in the literature.

A concrete explicit result presented in this work is the von Neumann equation for spin- $1 / 2$ system with generic stationary Hamiltonian written in the form of a system of kinetic equations for probability distributions identified with the system states. The solutions of this kinetic equations are given in the form of trajectories in the three-dimensional real space satisfying the system of linear equations for probabilities of spin projections on three perpendicular directions in the configuration space.

The problem of probability representation and symmetry of system evolutions related to non-Hermitian operators [39-42] needs further study.

The other result is the explicit form of a probabilistic realization of representation of Lie algebra, which determines the Heisenberg-Weyl symmetry group as well as the structure constants of associative product and Jordan product connected with this group. The results obtained can be extended to provide the probabilistic description of quantum states and their dynamics for systems with higher spins, qudits, and $N$-level atoms, as well as for two-mode photon states, using their higher symmetries.

The results obtained follow from the existence of an invertible map of the formalism of conventional description of quantum states by vectors and density operators in a Hilbert space onto probability distributions of random variables in the probability representation of quantum mechanics. The axioms of quantum mechanics $[3,5]$ and all discussed in the literature interpretation problems of quantum mechanics, including nonlocality and realism, can be also considered in the probability representation of quantum states. We point out that the probability representation considered in this work for studying examples of qubits and systems with continuous variables employs Born's rule, which is consistent with axioms and principles of quantum mechanics [3,5]. Namely Born's rule provides the possibility to construct the mentioned map of conventional Hilbert space formalism of quantum mechanics onto the formalism of probability distributions and their properties employed in the probability representation of quantum mechanics. If the dequantizer operators have the properties of density operators, the Born's rule applications provide the possibility to construct the map of the state density operators onto probability distributions identified with the quantum states.

It is worth mentioning some possible merits of our method in quantum information and quantum information processing. The method provides the possibility to use well-developed in probability theory information-entropic relations $[21,56]$ to obtain extra characteristics of quantum-state density matrices. Furthermore, in quantum information processing, gate operations can be formulated as linear 
transforms of probability distributions, for which some mathematical approach known in probability theory can be applied. We plan to investigate these aspects in future publications.

Author Contributions: Conceptualization, P.A. and V.I.M.; methodology, V.A.A. and M.A.M.; software, Matyas Mechler; validation, P.A. and V.A.A.; formal analysis, M.M.; investigation, M.A.M. and V.I.M.; writing-original draft preparation, P.A. and V.A.A.; writing-review and editing, M.A.M. All authors have read and agreed to the published version of the manuscript.

Funding: This research received no external funding.

Acknowledgments: This research was supported by the National Research, Development and Innovation Office, Hungary (Projects No. K124351 and No. 2017-1.2.1-NKP-2017-00001 HunQuTech). The project has also been supported by the European Union (Grants No. EFOP-3.6.2-16-2017-00005 and No. EFOP-3.4.3-16-2016-00005).

Conflicts of Interest: The authors declare no conflict of interest.

\section{References}

1. Schrödinger, E. Quantisierung als Eigenwertproblem (Erste Mitteilung). Ann. Phys. 1926, 384, 361-376, doi:10.1002/andp.19263840404. [CrossRef]

2. Schrödinger, E. Quantisierung als Eigenwertproblem (Zweite Mitteilung). Ann. Phys. 1926, 384, 489-527, doi:10.1002/andp.19263840602. [CrossRef]

3. Dirac, P.A.M. The Principles of Quantum Mechanics; Clarendon Press: Oxford, UK, 1981; ISBN 9780198520115.

4. Landau, L. Das Dämpfungsproblem in der Wellenmechanik. Z. Phys. 1927, 45, 430-441, doi:10.1007/BF01343064. [CrossRef]

5. Von Neumann, J. Wahrscheinlichkeitstheoretischer Aufbau der Quantenmechanik. Nachrichten von der Gesellschaft der Wissenschaften zu Gottingen Mathematisch-Physikalische Klasse 1927, 1927, 245-272.

6. Asorey, M.; Ibort, A.; Marmo, G.; Ventriglia, F. Quantum tomography twenty years later. Phys. Scr. 2015, 90, 074031, doi:10.1088/0031-8949/90/7/074031. [CrossRef]

7. Smithey, D.T.; Beck, M.; Raymer, M.G.; Faridani, A. Measurement of the Wigner distribution and the density matrix of a light mode using optical homodyne tomography: Application to squeezed states and the vacuum. Phys. Rev. Lett. 1993, 70, 1244-1248, doi:10.1103/PhysRevLett.70.1244. [CrossRef] [PubMed]

8. Lvovsky, A.I.; Raymer, M.G. Continuous-variable optical quantum-state tomography. Rev. Mod. Phys. 2009, 81, 299-332, doi:10.1103/RevModPhys.81.299. [CrossRef]

9. Radon, J.; Parks, P.; Clark, C. On the determination of functions from their integral values along certain manifolds. IEEE Trans. Med. Imaging 1986, 5, 170-176.

10. Bertrand, J.; Bertrand, P. A tomographic approach to Wigner's function. Found. Phys. 1987, 17, 397-405, doi:10.1007/BF00733376. [CrossRef]

11. Vogel, K.; Risken, H. Determination of quasiprobability distributions in terms of probability distributions for the rotated quadrature phase. Phys. Rev. A 1989, 40, 2847-2849. [CrossRef] [PubMed]

12. Mancini, S.; Man'ko, V.I.; Tombesi, P. Symplectic tomography as classical approach to quantum systems. Phys. Lett. A 1996, 213, 1-6, doi:10.1016/0375-9601(96)00107-7. [CrossRef]

13. Mancini, S.; Man'ko, V.I.; Tombest, P. Classical-like description of quantum dynamics by means of symplectic tomography. Found. Phys. 1997, 27, 801-824. [CrossRef]

14. Korennoy, Y.A.; Man'ko, V.I. Probability representation of the quantum evolution and energy-level equations for optical tomograms. J. Russ. Laser Res. 2011, 32, 74-95, doi:10.1007/s10946-011-9191-5. [CrossRef]

15. Amosov, G.G.; Korennoy, Y.A.; Man'ko, V.I. Description and measurement of observables in the optical tomographic probability representation of quantum mechanics. Phys. Rev. A 2012, 85, 052119, doi:10.1103/PhysRevA.85.052119. [CrossRef]

16. Dodonov, V.V.; Man'ko, V.I. Positive distribution description for spin states. Phys. Lett. A 1997, 229, 335-339, doi:10.1016/S0375-9601(97)00199-0. [CrossRef]

17. Man'ko, V.I.; Man'ko, O.V. Spin state tomography. J. Exp. Theor. Phys. 1997, 85, 430-434. [CrossRef]

18. Amiet, J.-P.; Weigert, S. Reconstructing a pure state of a spin $s$ through three Stern-Gerlach measurements. J. Phys. A Math. Gen. 1999, 32, 2777. [CrossRef]

19. Amiet, J.-P.; Weigert, S. Coherent states and the reconstruction of pure spin states. J. Opt. B Quantum Semiclass. Opt. 1999, 1, L5-L8, doi:10.1088/1464-4266/1/5/101. [CrossRef] 
20. D'Ariano, G.M.; Maccone, L.; Paini, M. Spin tomography. J. Opt. B Quantum Semiclass. Opt. 2003, 5, 77-84. [CrossRef]

21. Khrennikov, A. Probability and Randomness. Quantum versus Classical; World Scientific: Singapore, 2016; doi:10.1142/p1036. [CrossRef]

22. Man'ko, O.V.; Man'ko, V.I.; Marmo, G. Alternative commutation relations, star products and tomography. J. Phys. A Math. Gen. 2002, 35, 699-719. [CrossRef]

23. Ciaglia, F.M.; Di Cosmo, F.; Ibort, A.; Marmo, G. Dynamical aspects in the quantizer-dequantizer formalism. Ann. Phys. 2017, 385, 769-781. [CrossRef]

24. Terra-Cunha, M.O.; Man'ko, V.I.; Scully, M.O. Quasiprobability and probability distributions for spin-1/2 states. Found. Phys. Lett. 2001, 14, 103-117.:1012373419313. [CrossRef]

25. Man'ko, V.I.; Marmo, G.; Ventriglia, F.; Vitale, P. Metric on the space of quantum states from relative entropy. Tomographic reconstruction. J.Phys. A Math. Gen. 2017, 50, 335302. [CrossRef]

26. Wigner, E. On the quantum correction for thermodynamic equilibrium. Phys. Rev. 1932, 40, 749-759. [CrossRef]

27. Husimi, K. Some formal properties of the density matrix. Proc. Phys. Math. Soc. Jpn. 1940, 22, 264-314, doi:10.11429/ppmsj1919.22.4_264. [CrossRef]

28. Glauber, R.J. Coherent and incoherent states of the radiation field. Phys. Rev. 1963, 131, 2766-2788, doi:10.1103/PhysRev.131.2766. [CrossRef]

29. Sudarshan, E.C.G. Equivalence of semiclassical and quantum-mechanical descriptions of statistical light beams. Phys. Rev. Lett. 1963, 10, 277-279. [CrossRef]

30. Scully, M.O.; Wodkiewicz, K. Spin quasidistribution functions. Found. Phys. 1994, 24, 85-107. [CrossRef]

31. Stratonovich, R.L. On distributions in representation space. J. Exp. Theor. Phys. 1957, 4, 891-898,

32. Heisenberg, W. Uber den anschaulichen Inhalt der quantentheoretischen Kinematik und Mechanik. Z. Phys. 1927, 43, 172-198. [CrossRef]

33. Schrödinger, E. Zum Heisenbergschen Unscharfeprinzip; Berliner Königlich Akademie und die Wissenschaft: Berlin, Germany, 1930; pp. 296-303.

34. Robertson, H.R. The uncertainty principle. Phys. Rev. 1929, 34, 163-164. [CrossRef]

35. Klimov, A.B.; Romero, J.L.; Björk, G.; Sánchez-Soto, L.L. Geometrical approach to mutually unbiased bases. J. Phys. A Math. Gen. 2007, 40, 3987-3998. [CrossRef]

36. Lizzi, F.; Vitale, P. Matrix bases for star-products: A review. Symmetry Integr. Geom. Methods Appl. 2014, 10, 086, doi:10.3842/SIGMA.2014.086.4/014. [CrossRef]

37. Mancini, S.; Man'ko, O.V.; Man'ko, V.I.; Tombesi, P. The Pauli equation for probability distributions. J. Phys. A Math. Gen. 2001, 34, 3461-3476. [CrossRef]

38. Man'ko, M.A.; Man'ko, V.I. Observables, interference phenomenon and Born's rule in the probability representation of quantum mechanics. Int. J. Quantum Inform. 2020, 18, 1941021, doi:10.1142/S0219749919410211. [CrossRef]

39. Bender, C.M.; Boettcher, S. Real spectra in non-hermitian Hamiltonians having PT symmetry. Phys. Rev. Lett. 1998, 80, 5243-5246, doi:10.1103/PhysRevLett.80.5243. [CrossRef]

40. Mostafazadeh, A. Pseudo-Hermitian representation of quantum mechanics. Int. J. Geom. Methods Mod. Phys. 2010, 7, 1191-1306, doi:10.1142/S0219887810004816. [CrossRef]

41. Sergi, A.; Zloshchastiev, K.G. Non-Hermitian quantum dynamics of a two-level system and models of dissipative environments. Int. J. Mod. Phys. B 2013, 27, 1350163. [CrossRef]

42. Sergi, A.; Giaquinta, P.V. Linear quantum entropy and non-Hermitian Hamiltonians. Entropy 2016, 18, 451, doi:10.3390/e18120451. [CrossRef]

43. Kolmogorov, A.N. Foundation of the Theory of Probability; Chelsea: New York, NY, USA, 1956; ISBN 10:0821826484; ISBN 13 / 9780821826485.

44. Malkin, I.A.; Man'ko, V.I. Symmetry of the hydrogen atom. JETP Lett. 1965, 2, 146-148.

45. Barut, A.O.; Kleinert, H. Transition probabilities of the hydrogen atom from noncompact dynamical groups. Phys. Rev. 1967, 156, 1541-1545. [CrossRef]

46. Barut, A.; Bohm, A.; Neeman, Y. Dynamical Groups and Spectrum Generating Algebras; World Scientific: Singapore, 1986; ISBN 978-9971-5-0147-1, ISBN 9971-5-0147-3.

47. Andreev, V.A.; Malkin, I.A.; Man'ko, V.I. Dynamical Symmetries of Magnetic Monopole; Preprint No. 1; Lebedev Physical Institute: Moscow, Russia, 1971. 
48. Chernega, V.N.; Man'ko, O.V.; Man'ko, V.I. Triangle geometry of the qubit state in the probability representation expressed in terms of the Triada of Malevich's Squares. J. Russ. Laser Res. 2017, 38, 141-149, doi:10.1007/s10946-017-9628-6. [CrossRef]

49. Chernega, V.N.; Man'ko, O.V.; Man'ko, V.I. Probability representation of quantum observables and quantum states. J. Russ. Laser Res. 2017, 38, 324-333. [CrossRef]

50. Chernega, V.N.; Man'ko, O.V.; Man'ko, V.I. Triangle geometry for qutrit states in the probability representation. J. Russ. Laser Res. 2017, 38, 416-425. [CrossRef]

51. Sudarshan, E.C.G. Search for purity and entanglement. J. Russ. Laser Res. 2003, 24, 195-203.:1024090024283. [CrossRef]

52. Adam, P.; Andreev, V.A.; Isar, A.; Man'ko, M.A.; Man'ko, V.I. Continuous sets of dequantizers and quantizers for one-qubit states. J. Russ. Laser Res. 2016, 37, 544-555. 10.1007/s10946-016-9606-4. [CrossRef]

53. Adam, P.; Andreev, V.A.; Isar, A.; Man'ko, M.A.; Man'ko, V.I. Minimal sets of dequantizers and quantizers for finite-dimensional quantum systems. Phys. Lett. A 2017, 381, 2778-2782, doi:10.1016/j.physleta.2017.06.042. [CrossRef]

54. Adam, P.; Andreev, V.A.; Man'ko, M.A.; Man'ko, V.I. Nonnnegative discrete symbols and their probabilistic interpretation. J. Russ. Laser Res. 2017, 38, 491-506.10.1007/s10946-017-9673-1. [CrossRef]

55. Adam, P.; Andreev, V.A.; Man'ko, M.A.; Man'ko, V.I. Symbols of multiqubit states admitting a physical interpretation. J. Russ. Laser Res. 2018, 39, 360-375. 10.1007/s10946-018-9730-4. [CrossRef]

56. Man'ko, M.A.; Man'ko, V.I. New entropic inequalities and hidden correlations in quantum suprematism picture of qudit states. Entropy 2018, 20, 692. [CrossRef]

(C) 2020 by the authors. Licensee MDPI, Basel, Switzerland. This article is an open access article distributed under the terms and conditions of the Creative Commons Attribution (CC BY) license (http:/ / creativecommons.org/licenses/by/4.0/). 